\title{
COMPARISON OF DIFFERENCES BETWEEN ARITHMETIC AND GEOMETRIC MEANS
}

\author{
J. M. ALDAZ
}

Abstract. We complement a recent result of S. Furuichi, by showing that the differences $\sum_{i=1}^{n} \alpha_{i} x_{i}-\prod_{i=1}^{n} x_{i}^{\alpha_{i}}$ associated to distinct sequences of weights are comparable, with constants that depend on the smallest and largest quotients of the weights.

\section{Introduction}

It is well known that the inequality between arithmetic and geometric means is selfimproving, that is, increasingly better versions of the AM-GM inequality can be obtained simply by repeated applications of itself. The simplest case of the inequality, $\sqrt{x y} \leq(x+y) / 2$, is trivial and equivalent to $(\sqrt{x}-\sqrt{y})^{2} \geq 0$. By repeated self-improvement (cf. [11] for more details) it is possible to obtain from $(\sqrt{x}-\sqrt{y})^{2} \geq 0$ the general AM-GM inequality

$$
\prod_{i=1}^{n} x_{i}^{\alpha_{i}} \leq \sum_{i=1}^{n} \alpha_{i} x_{i}
$$

Of course, there are more efficient ways to prove (1). Nevertheless, the idea of self-improvement is a useful one: It has recently been utilized to find refinements of the AM-GM inequality, and to give better proofs of existing refinements. For instance, self-improvement via the change of variables $x_{i}=y_{i}^{s}$, was used in [1], with $s=1 / 2$, to show that

$$
\sum_{i=1}^{n} \alpha_{i} x_{i}-\prod_{i=1}^{n} x_{i}^{\alpha_{i}} \geq \sum_{i=1}^{n} \alpha_{i}\left(x_{i}^{1 / 2}-\sum_{k=1}^{n} \alpha_{k} x_{k}^{1 / 2}\right)^{2} .
$$

Observe that the right hand side of (2) is the variance $\operatorname{Var}\left(x^{1 / 2}\right)$ of the vector $x^{1 / 2}=\left(x_{1}^{1 / 2}, \ldots, x_{n}^{1 / 2}\right)$ with respect to the discrete probability $\sum_{i=1}^{n} \alpha_{i} \delta_{x_{i}}$. While a large variance (of $x^{1 / 2}$ ) pushes the arithmetic and geometric means apart, no conclusions can be derived from a small variance, as noted in [1]. Suppose $n>>1$ and $x_{1}=\cdots=x_{n}$, so $\operatorname{Var}\left(x^{1 / 2}\right)=0$. If $\alpha_{j}$ is the smallest weight, letting $x_{j} \downarrow 0$ leaves $\operatorname{Var}\left(x^{1 / 2}\right)$ and $\sum_{i=1}^{n} \alpha_{i} x_{i}$ essentially unchanged, while $\prod_{i=1}^{n} x_{i}^{\alpha_{i}}$ drops to 0 .

2010 Mathematics Subject Classification. 26D15.

Key words and phrases. Self-improvement, arithmetic-geometric inequality. Partially supported by Grant MTM2009-12740-C03-03 of the D.G.I. of Spain. 
Thus, an upper bound for $\sum_{i=1}^{n} \alpha_{i} x_{i}-\prod_{i=1}^{n} x_{i}^{\alpha_{i}}$ in terms of $\operatorname{Var}\left(x^{1 / 2}\right)$ alone cannot be found, so it is natural to seek an alternative way to control this difference.

Using an idea originally due to S. Dragomir (cf. [5]), S. Furuichi showed by self-improvement that $\sum_{i=1}^{n} \alpha_{i} x_{i}-\prod_{i=1}^{n} x_{i}^{\alpha_{i}} \geq n \alpha_{\min }\left(n^{-1} \sum_{i=1}^{n} x_{i}-\prod_{i=1}^{n} x_{i}^{1 / n}\right)$, where $\alpha_{\min }:=\min \left\{\alpha_{1}, \ldots, \alpha_{n}\right\}$, cf. [8]. This result generalizes one side of the two-sided refinement of Young's inequality presented in [4, Lemma 2.1]. We show in this note that the equal weights AM-GM difference can be utilized to give an entirely analogous upper bound, with $\alpha_{\max }:=\max \left\{\alpha_{1}, \ldots, \alpha_{n}\right\}$ replacing $\alpha_{\text {min }}$. More generally, we shall see that differences between arithmetic and geometric means associated to different sequences of weights are comparable, with constants depending on the maxima and minima of the sequence of quotients of weights. A standard application to Hölder's inequality is presented next. We finish with a discussion regarding the "typical size", in a certain probabilistic sense, of $\sum_{i=1}^{n} \alpha_{i} x_{i}-\prod_{i=1}^{n} x_{i}^{\alpha_{i}}$ and of $\prod_{i=1}^{n} x_{i}^{\alpha_{i}} / \sum_{i=1}^{n} \alpha_{i} x_{i}$ when $n>>1$.

\section{Self-bounds on AM-GM differences}

The first version of this note was written without the author being aware of [5]. Originally, Theorem 2.1 had weaker bounds, given in terms of $\alpha_{\min } / \beta_{\max }$ and $\alpha_{\max } / \beta_{\min }$, where $\alpha_{\min }:=$ $\min \left\{\alpha_{1}, \ldots, \alpha_{n}\right\}, \alpha_{\max }:=\max \left\{\alpha_{1}, \ldots, \alpha_{n}\right\}$, and analogously for $\beta_{\min }$ and $\beta_{\max }$. It was pointed out by an anonymous referee that the stronger bounds appearing in (3) could be obtained by applying [5, Theorem 1] (which refines Jensen's inequality) to the function $f(t)=e^{t}$. This also has been observed by Flavia Corina Mitroi (personal communication, cf. [10]).

Here we note that the same self-improvement argument used by S. Dragomir in [5], yields (3) directly from the AM-GM inequality. We also study the equality conditions, which are not considered in [5].

Theorem 2.1. For $n \geq 2$ and $i=1, \ldots, n$, let $x_{i} \geq 0$, and let $\alpha_{i}, \beta_{i}>0$ satisfy $\sum_{i=1}^{n} \alpha_{i}=\sum_{i=1}^{n} \beta_{i}=$ 1. Then we have

$$
\min _{k=1, \ldots, n}\left\{\frac{\alpha_{k}}{\beta_{k}}\right\}\left(\sum_{i=1}^{n} \beta_{i} x_{i}-\prod_{i=1}^{n} x_{i}^{\beta_{i}}\right) \leq \sum_{i=1}^{n} \alpha_{i} x_{i}-\prod_{i=1}^{n} x_{i}^{\alpha_{i}} \leq \max _{k=1, \ldots, n}\left\{\frac{\alpha_{k}}{\beta_{k}}\right\}\left(\sum_{i=1}^{n} \beta_{i} x_{i}-\prod_{i=1}^{n} x_{i}^{\beta_{i}}\right) .
$$

Regarding the equality conditions, set $A:=\left\{i: \alpha_{i} / \beta_{i}=\min \left\{\alpha_{k} / \beta_{k}: 1 \leq k \leq n\right\}, i=1, \ldots, n\right\}$ and $B=\left\{i: \alpha_{i} / \beta_{i}=\max \left\{\alpha_{k} / \beta_{k}: 1 \leq k \leq n\right\}, i=1, \ldots, n\right\}$. Then we have equality on the left hand side of (3) if and only iffor every $j \in\{1, \cdots, n\} \backslash A$,

$$
x_{j}=\left(\prod_{i \in A} x_{i}^{\alpha_{i}}\right)^{\frac{1}{\sum_{i \in A} \alpha_{i}}}=\prod_{i=1}^{n} x_{i}^{\alpha_{i}}, \text { or equivalently, } x_{j}=\left(\prod_{i \in A} x_{i}^{\beta_{i}}\right)^{\frac{1}{\sum_{i \in A} \beta_{i}}}=\prod_{i=1}^{n} x_{i}^{\beta_{i}},
$$


while equality holds on the right hand side of (3) if and only if for every $j \in\{1, \cdots, n\} \backslash B$,

$$
x_{j}=\left(\prod_{i \in B} x_{i}^{\alpha_{i}}\right)^{\frac{1}{\sum_{i \in B} \alpha_{i}}}=\prod_{i=1}^{n} x_{i}^{\alpha_{i}} \text {, or equivalently, } x_{j}=\left(\prod_{i \in B} x_{i}^{\beta_{i}}\right)^{\frac{1}{\sum_{i \in B} \beta_{i}}}=\prod_{i=1}^{n} x_{i}^{\beta_{i}} .
$$

Let $|A|$ and $|B|$ denote the cardinalities of the sets $A$ and $B$. Observe that when $|A|=1$, equality holds on the left hand side of (3) if and only if $x_{1}=\cdots=x_{n}$, and likewise for the right hand side when $|B|=1$.

Before proving Theorem 2.1, we present an example illustrating the need for the slightly complicated formulation of the equality conditions (which were incorrectly stated in the first version of this note, after reading [8] without enough care). We want to stress the fact that the left and right hand sides of (3) have to be dealt with separately.

Example 2.2. In formula (3), let $n=3, \alpha_{1}=2 / 3, \alpha_{2}=\alpha_{3}=1 / 6$, and for $i=1,2,3, \beta_{i}=1 / 3$. As a normalization, suppose also that $(x y z)^{1 / 3}=1$. Then $|B|=1$, and equality holds on the right hand side of (3) if and only if $x=y=z=1$. However, this condition is too strong for the left hand side since $1<|A|=2$; there, equality holds if and only if $x=y z=1$.

Proof. The second inequality in (3) is equivalent to

$$
\prod_{i=1}^{n} x_{i}^{\beta_{i}} \leq \sum_{i=1}^{n}\left(\beta_{i}-\min _{k=1, \ldots, n}\left\{\frac{\beta_{k}}{\alpha_{k}}\right\} \alpha_{i}\right) x_{i}+\min _{k=1, \ldots, n}\left\{\frac{\beta_{k}}{\alpha_{k}}\right\} \prod_{i=1}^{n} x_{i}^{\alpha_{i}} .
$$

Writing

$$
\prod_{i=1}^{n} x_{i}^{\beta_{i}}=\prod_{i=1}^{n} x_{i}^{\beta_{i}-\min _{k=1, \ldots, n}\left\{\frac{\beta_{k}}{\alpha_{k}}\right\} \alpha_{i}} \prod_{i=1}^{n} x_{i}^{\min _{k=1, \ldots, n}\left\{\frac{\beta_{k}}{\alpha_{k}}\right\} \alpha_{i},}
$$

we see that (6) is just the AM-GM inequality, since $0=\beta_{i}-\frac{\beta_{i}}{\alpha_{i}} \alpha_{i} \leq \beta_{i}-\min _{k=1, \ldots, n}\left\{\frac{\beta_{k}}{\alpha_{k}}\right\} \alpha_{i}$ and

$$
\sum_{i=1}^{n}\left(\beta_{i}-\min _{k=1, \ldots, n}\left\{\frac{\beta_{k}}{\alpha_{k}}\right\} \alpha_{i}\right)+\min _{k=1, \ldots, n}\left\{\frac{\beta_{k}}{\alpha_{k}}\right\}=1
$$

To obtain the first inequality in formula (3), multiply both sides of the second inequality by $\min _{k=1, \ldots, n}\left\{\frac{\beta_{k}}{\alpha_{k}}\right\}$, and note that this is just the first inequality with the roles of the $\alpha$ 's and the $\beta$ 's interchanged. Alternatively, it is possible to prove the first inequality directly, using the same argument as above, and derive the second from the first.

For the case of equality, set $q_{i}:=\alpha_{i} / \beta_{i}$ and suppose that $q_{1} \leq \cdots \leq q_{n}$ (by rearranging the sequences of weights, if needed). If $r:=|A|=n$, then also $s:=|B|=n$, all $q_{i}=1$, and thus, for $i=1, \ldots, n, \alpha_{i}=\beta_{i}$, whence equality trivially holds on both sides of (3), without any conditions imposed on the variables $x_{i}$. If $r<n$, then $r<n-s+1 \leq n$, and $q_{n-s}<q_{n-s+1}=\cdots=q_{n}$. Assume that equality holds on the right hand side of (3), or, equivalently, that

$$
\prod_{i=1}^{n} x_{i}^{\beta_{i}}=\sum_{i=1}^{n}\left(\beta_{i}-\min _{k=1, \ldots, n}\left\{\frac{\beta_{k}}{\alpha_{k}}\right\} \alpha_{i}\right) x_{i}+\min _{k=1, \ldots, n}\left\{\frac{\beta_{k}}{\alpha_{k}}\right\} \prod_{i=1}^{n} x_{i}^{\alpha_{i}} .
$$


Removing the zero terms from the preceding sum, we see that (8) holds if and only if

$$
\sum_{i=1}^{n-s}\left(\beta_{i}-\min _{k=1, \ldots, n}\left\{\frac{\beta_{k}}{\alpha_{k}}\right\} \alpha_{i}\right) x_{i}+\min _{k=1, \ldots, n}\left\{\frac{\beta_{k}}{\alpha_{k}}\right\} \prod_{i=1}^{n} x_{i}^{\alpha_{i}}=\prod_{i=1}^{n} x_{i}^{\beta_{i}}
$$

It now follows from the equality condition in the AMGM inequality that (9) holds if and only if

$$
c:=x_{1}=\cdots=x_{n-s}=\prod_{i=1}^{n} x_{i}^{\alpha_{i}}=\prod_{i=1}^{n} x_{i}^{\beta_{i}} .
$$

Thus,

$$
c=c^{\sum_{i=1}^{n-s} \alpha_{i}} \prod_{i=n-s+1}^{n} x_{i}^{\alpha_{i}}=c^{\sum_{i=1}^{n-s} \beta_{i}} \prod_{i=n-s+1}^{n} x_{i}^{\beta_{i}},
$$

and we obtain the conditions appearing in (4) by simplifying and solving for $c$. To see that one of these conditions is redundant, recall that for $i=n-s+1, \ldots, n, \beta_{i}=\min _{k=1, \ldots, n}\left\{\frac{\beta_{k}}{\alpha_{k}}\right\} \alpha_{i}$, so

$$
\left(\prod_{i=n-s+1}^{n} x_{i}^{\alpha_{i}}\right)^{\frac{1}{\sum_{i=n-s+1}^{n} \alpha_{i}}}=\left(\prod_{i=n-s+1}^{n} x_{i}^{\beta_{i}}\right)^{\frac{1}{\sum_{i=n-s+1}^{n} \beta_{i}}} .
$$

The equality conditions for the left hand side of (3) can be obtained in the same way, or, alternatively, from those for the right hand side, by interchanging the roles of the $\alpha$ 's and $\beta$ 's.

Recall that $\alpha_{\min }:=\min \left\{\alpha_{1}, \ldots, \alpha_{n}\right\}$ and $\alpha_{\max }:=\max \left\{\alpha_{1}, \ldots, \alpha_{n}\right\}$.

Corollary 2.3. Under the same hypotheses and with the notation of Theorem 2.1, let $\beta_{i}=1 / n$ for all $i=1, \ldots, n$. Then

$$
n \alpha_{\min }\left(\frac{1}{n} \sum_{i=1}^{n} x_{i}-\prod_{i=1}^{n} x_{i}^{1 / n}\right) \leq \sum_{i=1}^{n} \alpha_{i} x_{i}-\prod_{i=1}^{n} x_{i}^{\alpha_{i}} \leq n \alpha_{\max }\left(\frac{1}{n} \sum_{i=1}^{n} x_{i}-\prod_{i=1}^{n} x_{i}^{1 / n}\right) .
$$

The left hand side of (12) is essentially the content of [8]. For completeness, next we consider the equality case in the Dragomir-Jensen inequality for strictly convex functions.

Proposition 2.4. For $n \geq 2$ and $i=1, \ldots, n$, let $\alpha_{i}, \beta_{i}>0$ satisfy $\sum_{i=1}^{n} \alpha_{i}=\sum_{i=1}^{n} \beta_{i}=1$. Let $f: C \rightarrow \mathbb{R}$ be strictly convex, where $C$ is a convex subset of a linear space. As in Theorem 2.1, we set $A:=\left\{i: \alpha_{i} / \beta_{i}=\min \left\{\alpha_{k} / \beta_{k}: 1 \leq k \leq n\right\}, i=1, \ldots, n\right\}$ and $B=\left\{i: \alpha_{i} / \beta_{i}=\max \left\{\alpha_{k} / \beta_{k}\right.\right.$ : $1 \leq k \leq n\}, i=1, \ldots, n\}$. Then, we have equality on the left hand side of the Dragomir-Jensen inequalities

$$
\begin{gathered}
\min _{k=1, \ldots, n}\left\{\frac{\alpha_{k}}{\beta_{k}}\right\}\left(\sum_{i=1}^{n} \beta_{i} f\left(x_{i}\right)-f\left(\sum_{i=1}^{n} \beta_{i} x_{i}\right)\right) \leq \\
\sum_{i=1}^{n} \alpha_{i} f\left(x_{i}\right)-f\left(\sum_{i=1}^{n} \alpha_{i} x_{i}\right) \leq \max _{k=1, \ldots, n}\left\{\frac{\alpha_{k}}{\beta_{k}}\right\}\left(\sum_{i=1}^{n} \beta_{i} f\left(x_{i}\right)-f\left(\sum_{i=1}^{n} \beta_{i} x_{i}\right)\right)
\end{gathered}
$$


if and only iffor every $j \in\{1, \cdots, n\} \backslash A$,

$$
x_{j}=\sum_{i \in A} \frac{\alpha_{i}}{\sum_{i \in A} \alpha_{i}} x_{i}=\sum_{i=1}^{n} \alpha_{i} x_{i} \text {, or equivalently, } x_{j}=\sum_{i \in A} \frac{\beta_{i}}{\sum_{i \in A} \beta_{i}} x_{i}=\sum_{i=1}^{n} \beta_{i} x_{i},
$$

while equality holds on the right hand side of (13)-(14) if and only if for every $j \in\{1, \cdots, n\} \backslash B$,

$$
x_{j}=\sum_{i \in B} \frac{\alpha_{i}}{\sum_{i \in B} \alpha_{i}} x_{i}=\sum_{i=1}^{n} \alpha_{i} x_{i} \text {, or equivalently, } x_{j}=\sum_{i \in B} \frac{\beta_{i}}{\sum_{i \in B} \beta_{i}} x_{i}=\sum_{i=1}^{n} \beta_{i} x_{i} .
$$

Proof. As in the proof of Theorem 2.1, we set $q_{i}:=\alpha_{i} / \beta_{i}$ and suppose that $q_{1} \leq \cdots \leq q_{n}$. If $r:=|A|=n$, then $\alpha_{i}=\beta_{i}$ for all $i=1, \ldots, n$, and equality trivially holds on both (13) and (14), without any conditions on the $x_{i}$ 's (and without needing the strict convexity of $f$ ). If $r<n$, then $r<n-s+1 \leq n$, and $q_{n-s}<q_{n-s+q}=\cdots=q_{n}$. Assume that equality holds on (14), or, equivalently, after removing the zero summands, that

$$
f\left(\sum_{i=1}^{n} \beta_{i} x_{i}\right)=\sum_{i=1}^{n-s}\left(\beta_{i}-\min _{k=1, \ldots, n}\left\{\frac{\beta_{k}}{\alpha_{k}}\right\} \alpha_{i}\right) f\left(x_{i}\right)+\min _{k=1, \ldots, n}\left\{\frac{\beta_{k}}{\alpha_{k}}\right\} f\left(\sum_{i=1}^{n} \alpha_{i} x_{i}\right) .
$$

Using the equality condition for strictly convex functions in Jensen's inequality, we see that (17) holds if and only if

$$
c:=x_{1}=\cdots=x_{n-s}=\sum_{i=1}^{n} \alpha_{i} x_{i}=\sum_{i=1}^{n} \beta_{i} x_{i} .
$$

Replacing in the preceding sums $x_{i}$ by $c$ for $1 \leq i \leq n-s$, we conclude that (18) holds if and only if

$$
c=\sum_{i=n-s+1}^{n} \frac{\alpha_{i}}{\sum_{i=n-s+1}^{n} \alpha_{i}} x_{i}
$$

or equivalently, if and only if

$$
c=\sum_{i=n-s+1}^{n} \frac{\beta_{i}}{\sum_{i=n-s+1}^{n} \beta_{i}} x_{i} .
$$

The proof for the left hand side of (13)-(14) is entirely analogous; it can also be obtained from the right hand side.

\section{Refinements of Hölder's inequality}

When the AM-GM inequality is specialized to just two terms, it is usually called Young's inequality. Next we utilize the preceding results to generalize [4, Lemma 2.1] and [4, Theorem 2.2], giving two-sided refinements of Hölder's inequality for two functions. When considering $L^{p}=L^{p}(\mu)$ spaces, we always assume that $\mu$ is not identically zero. 
Corollary 3.1. Let $1<p<\infty$ and let $q=p /(p-1)$ be its conjugate exponent. Then for all $u, v \geq 0$ and all $\beta \in(0,1)$,

$$
\begin{gathered}
\min \left\{\frac{1}{\beta p}, \frac{1}{(1-\beta) q}\right\}\left(\beta u^{p}+(1-\beta) v^{q}-u^{\beta p} v^{(1-\beta) q}\right) \leq \frac{u^{p}}{p}+\frac{v^{q}}{q}-u v \\
\leq \max \left\{\frac{1}{\beta p}, \frac{1}{(1-\beta) q}\right\}\left(\beta u^{p}+(1-\beta) v^{q}-u^{\beta p} v^{(1-\beta) q}\right) .
\end{gathered}
$$

Proof. Set $n=2, \alpha=1 / p, 1-\alpha=1 / q, x_{1}=u^{p}$ and $x_{2}=v^{q}$ in Theorem 2.1.

Theorem 3.2. Let $1<p<\infty$, and let $q=p /(p-1)$ be its conjugate exponent. If $f \in L^{p}, g \in L^{q}$, $f, g \geq 0$, and $\|f\|_{p},\|g\|_{q}>0$, then for all $\beta \in(0,1)$,

$$
\begin{gathered}
\|f\|_{p}\|g\|_{q}\left(1-\max \left\{\frac{1}{\beta p}, \frac{1}{(1-\beta) q}\right\}\left(1-\frac{\int f^{\beta p} g^{(1-\beta) q}}{\left(\int f^{p}\right)^{\beta}\left(\int g^{q}\right)^{1-\beta}}\right)\right) \leq\|f g\|_{1} \\
\leq\|f\|_{p}\|g\|_{q}\left(1-\min \left\{\frac{1}{\beta p}, \frac{1}{(1-\beta) q}\right\}\left(1-\frac{\int f^{\beta p} g^{(1-\beta) q}}{\left(\int f^{p}\right)^{\beta}\left(\int g^{q}\right)^{1-\beta}}\right)\right) .
\end{gathered}
$$

Proof. The standard derivation of Hölder's inequality from Young's inequality is applicable: Write $u=f(x) /\|f\|_{p}$ and $v=g(x) /\|g\|_{q}$ in (21)-(22), integrate and reorganize terms. The rearranging of terms is justified, since it follows from the proof that $\int f^{\beta p} g^{(1-\beta) q} \leq\left(\int f^{p}\right)^{\beta}\left(\int g^{q}\right)^{1-\beta}$ $<\infty$, so all the quantities involved in (23) and (24) are finite (alternatively, since $f^{\beta p} \in$ $L^{1 / \beta}$ and $g^{(1-\beta) q} \in L^{1 /(1-\beta)}$, the bound $\int f^{\beta p} g^{(1-\beta) q}<\infty$ can also be deduced from Hölder's inequality).

Observe that setting $\beta=1 / p$ in (23) and (24), the preceding inequalities become trivial equalities, as was to be expected from the equality conditions in Theorem 2.1. When $\beta=1 / 2$, the formulas in (23) and (24) can be expressed using the angular distance $\left\|\frac{f^{p / 2}}{\|f\|_{p}^{p / 2}}-\frac{g^{q / 2}}{\|g\|_{q}^{q / 2}}\right\|_{2}$ between the $L^{2}$ functions $f^{p / 2}$ and $g^{q / 2}$ (cf. [4, Theorem 2.2]) so if the the angular distance is "large", then $\|f g\|_{1}$ is "small", and viceversa. We are not aware of any simple geometric interpretation of the bounds in (23)-(24) when $\beta \neq 1 / 2$.

We finish this section by stating the corresponding refinement of Hölder's inequality for several functions, in the simplest case $\beta_{i}=1 / n$ (of course, other values of $\beta_{i}$ can be used if it is convenient). The proof is standard and therefore ommited.

Theorem 3.3. For $i=1, \ldots, n$, let $1<p_{i}<\infty$ be such that $p_{1}^{-1}+\cdots+p_{n}^{-1}=1$, and let $0 \leq f_{i} \in L^{p_{i}}$ satisfy $\left\|f_{i}\right\|_{p_{i}}>0$. Writting $p_{\min }^{-1}=\min \left\{p_{1}^{-1}, \ldots, p_{n}^{-1}\right\}$ and $p_{\max }^{-1}=\max \left\{p_{1}^{-1}, \ldots, p_{n}^{-1}\right\}$, we have

$$
\prod_{i=1}^{n}\left\|f_{i}\right\|_{p_{i}}\left(1-n p_{\max }^{-1}\left(1-\frac{\int \prod_{i=1}^{n} f_{i}^{p_{i} / n}}{\prod_{i=1}^{n}\left\|f_{i}\right\|_{p_{i}}^{p_{i} / n}}\right)\right) \leq\left\|\prod_{i=1}^{n} f_{i}\right\|_{1}
$$




$$
\leq \prod_{i=1}^{n}\left\|f_{i}\right\|_{p_{i}}\left(1-n p_{\min }^{-1}\left(1-\frac{\int \prod_{i=1}^{n} f_{i}^{p_{i} / n}}{\prod_{i=1}^{n}\left\|f_{i}\right\|_{p_{i}}^{p_{i} / n}}\right)\right) .
$$

\section{Probabilistic considerations}

By comparability of AM-GM differences, known results about the typical behavior of the AM-GM inequality in the equal weights case, can be used to give bounds for other sequences of weights on the same probability spaces. Given $n \geq 2$ and $x=\left(x_{1}, \ldots, x_{n}\right) \in \mathbb{R}^{n} \backslash\{0\}$, the equal weights GM-AM ratio is

$$
r_{n}(x):=\frac{\prod_{i=1}^{n}\left|x_{i}\right|^{1 / n}}{n^{-1} \sum_{i=1}^{n}\left|x_{i}\right|} .
$$

By the AM-GM inequality we know that $0 \leq r_{n}(x) \leq 1$ always. If each $x_{i}$ is chosen independently from $[0, \infty)$ according to a fixed exponential distribution, i.e., with probability density function $f_{\lambda}(t)=\lambda e^{-\lambda t}$, then by [9, Theorem 5.1], with probability 1

$$
\lim _{n \rightarrow \infty} r_{n}(x)=e^{-\gamma}
$$

where $\gamma$ is Euler's constant and $e^{-\gamma} \approx 0.5615$. Observe that the limit does not in any way depend on the parameter $\lambda$. The following related result appears in [3, Corollary 2.2]: Denoting by $\mathbb{S}_{1}^{n-1}$ the $\ell_{1}^{n}$ unit sphere in $\mathbb{R}^{d}$, i.e., $\mathbb{S}_{1}^{n-1}=\left\{x \in \mathbb{R}^{d}:\left|x_{1}\right|+\cdots+\left|x_{n}\right|=1\right\}$, we have

Theorem 4.1. Let $k, \varepsilon>0$, and let $P_{1}^{n-1}$ be the uniform probability on $\mathbb{S}_{1}^{n-1}$. Then there exists an $N=N(k, \varepsilon)$ such that for every $n \geq N$,

$$
P_{1}^{n-1}\left\{(1-\varepsilon) e^{-\gamma}<r_{n}(x)<(1+\varepsilon) e^{-\gamma}\right\} \geq 1-\frac{1}{n^{k}} .
$$

Next we explain why the notions of random choice in the above results are equivalent (as mentioned in [3, Remark 2.4]). Observe that $r_{n}(x)$ is homogeneous of degree zero, that is, constant on the rays $t x$, where $t>0, x \neq 0$. In particular, taking $t^{-1}=\sum_{i=1}^{n}\left|x_{i}\right|$, we may assume that $\sum_{i=1}^{n}\left|x_{i}\right|=1$, or equivalently, that $x \in \mathbb{S}_{1}^{n-1}$. It is then natural to define random choice by taking normalized area on $\mathbb{S}_{1}^{n-1}$ as our probability measure. Suppose next that we select points from the whole space $\mathbb{R}^{n}$, according to an exponential density $2^{-n} \lambda^{n} e^{-\lambda\|x\|_{1}}$ on $\mathbb{R}^{n}$ for some fixed $\lambda>0$, or equivalently, $\lambda^{n} e^{-\lambda\|x\|_{1}}$ on the positive cone $[0, \infty)^{n}$. Of course, an exponential distribution gives a larger probability to "small" vectors than to large vectors, but this has no effect on the result by zero homogeneity, and for the same reason, it does not make any difference which $\lambda>0$ we select. While all of this is intuitively obvious, for completeness we present the formal argument. 
Proposition 4.2. Let $P_{1}^{n-1}$ be the uniform probability on $\mathbb{S}_{1}^{n-1}$, and set $d P_{n}:=2^{-n} \lambda^{n} e^{-\lambda\|x\|_{1}} d x$, where $\lambda>0$. Then for every $u \in \mathbb{R}, P_{n}\left(\left\{r_{n}>u\right\}\right)=P_{1}^{n-1}\left(\left\{r_{n}>u\right\}\right)$.

Let us recall the coarea formula (for additional information cf. [7], pp. 248-250, or [6], pp. 117-119):

$$
\int_{\mathbb{R}^{n}} g(x)|J f(x)| d x=\int_{\mathbb{R}} \int_{\left\{f^{-1}(t)\right\}} g(x) d \mathscr{H}^{n-1}(x) d t .
$$

Here $f$ is assumed to be Lipschitz, $\mathscr{H}^{n-1}$ is the $n-1$ dimensional Hausdorff measure, and $|J f(x)|:=\sqrt{\operatorname{det} d f(x) d f(x)^{t}}$ denotes the modulus of the Jacobian.

Proof. It is well known and easy to check that the volume of the $\left(\mathbb{R}^{n},\|\cdot\|_{1}\right)$-unit ball is $\left|\mathbb{B}_{1}^{n}\right|=$ $2^{n} / n !$. The area $\left|\mathbb{S}_{1}^{n-1}\right|=\mathscr{H}^{n-1}\left(\mathbb{S}_{1}^{n-1}\right)$ of the $\ell_{1}$ unit sphere then follows from the coarea formula: For every $x \in \mathbb{R}^{n} \backslash \cup_{i=1}^{n}\left\{x_{i} \neq 0\right\}$, the function $f(x)=\|x\|_{1}$ is differentiable, and

$$
|J f(x)|=\sqrt{\operatorname{det}\left(d f(x) d f(x)^{t}\right)}=\sqrt{\sum_{i=1}^{n} 1}=\sqrt{n}
$$

a.e. on $\mathbb{R}^{n}$. Set $g(x)=\chi_{\mathbb{B}_{1}^{n}} /|J f(x)|$ in (30), and denote by $\mathbb{S}_{1}^{n-1}(\rho)$ the sphere centered at 0 of radius $\rho$ (when $\rho=1$ we omit it). Then

$$
\frac{2^{n}}{n !}=\left|\mathbb{B}_{1}^{n}\right|=\int_{\mathbb{B}_{1}^{n}} d x=\int_{0}^{1} \int_{\mathbb{S}_{1}^{n-1}(\rho)} \frac{1}{\sqrt{n}} d \mathscr{H}^{n-1}(x) d \rho=\frac{\left|\mathbb{S}_{1}^{n-1}\right|}{\sqrt{n}} \int_{0}^{1} \rho^{n-1} d \rho=\frac{\left|\mathbb{S}_{1}^{n-1}\right|}{n \sqrt{n}},
$$

so $\left|\mathbb{S}_{1}^{n-1}\right|=2^{n} \sqrt{n} / \Gamma(n)$. Observe that $P_{1}^{n-1}(A)=\mathscr{P}^{n-1}\left(A \cap \mathbb{S}_{1}^{n-1}\right) /\left|\mathbb{S}_{1}^{n-1}\right|$.

Recalling that the ratio $r_{n}$ is homogeneous of degree 0 , so $r_{n}(x)=r_{n}\left(x /\|x\|_{1}\right)$, we next set $g(x)=\chi_{\left\{r_{n}>u\right\}}(x) \exp \left(-\lambda \sum_{i=1}^{n}\left|x_{i}\right|\right) /|J f(x)|$ in (30). Since $|J f(x)|=\sqrt{n}$ a.e.,

$$
\begin{gathered}
P_{n}\left(\left\{r_{n}>u\right\}\right)=\frac{\lambda^{n}}{2^{n}} \int_{\mathbb{R}^{n}} \chi_{\left\{r_{n}>u\right\}}(x) \exp \left(-\lambda \sum_{i=1}^{n}\left|x_{i}\right|\right) d x \\
=\frac{\lambda^{n}}{2^{n} \sqrt{n}} \int_{0}^{\infty} \int_{\left\{\|x\|_{1}=t\right\}} \chi_{\left\{r_{n}>u\right\}}(x) e^{-\lambda t} d \mathscr{H}^{n-1}(x) d t \\
=\frac{\lambda^{n}}{2^{n} \sqrt{n}} \int_{0}^{\infty} e^{-\lambda t} t^{n-1} \int_{\left\{\|x\|_{1}=1\right\}} \chi_{\left\{r_{n}>u\right\}}(x) d \mathscr{P}^{n-1}(x) d t \\
=\frac{\Gamma(n)}{2^{n} \sqrt{n}} \int_{\mathbb{S}_{1}^{n-1}} \chi_{\left\{r_{n}>u\right\}}(x) d \mathscr{P}^{n-1}(x)=P_{1}^{n-1}\left(\left\{r_{n}>u\right\}\right) .
\end{gathered}
$$

The next result is stated in terms of independent choices from an exponential distribution on $\left[0, \infty\right.$ ) rather than on $\mathbb{R}$ (so we can write $x_{i}$ instead of $\left|x_{i}\right|$, and thus $\|x\|_{1}=\sum_{i=1}^{n} x_{i}$ ). As usual, the weights $\alpha_{i, n}>0$ are assumed to satisfy $\sum_{i=1}^{n} \alpha_{i, n}=1$, and the largest and smallest such weights are denoted by $\alpha_{\max , n}$ and $\alpha_{\min , n}$ respectively. We suppose that for each $n \geq 2$ we are given a sequence of weights $\left\{\alpha_{1, n}, \ldots, \alpha_{n, n}\right\}$. 
Theorem 4.3. Let $k, \lambda, \varepsilon>0$, and for $1 \leq i \leq n$, let $x_{i} \in[0, \infty)$ be chosen independently according to an exponential distribution with parameter $\lambda$. Let $P_{n}$ denote the product probability on $[0, \infty)^{n}$ with density $\lambda^{n} e^{-\lambda\|x\|_{1}}$. Then there exists an $N=N(k, \varepsilon)$ such that for every $n \geq N$,

$$
\begin{gathered}
P_{n}\left\{\left[1-(1+\varepsilon) e^{-\gamma}\right] \alpha_{\min , n}\|x\|_{1}<\sum_{i=1}^{n} \alpha_{i, n} x_{i}-\prod_{i=1}^{n} x_{i}^{\alpha_{i, n}}<\left[1-(1-\varepsilon) e^{-\gamma}\right] \alpha_{\max , n}\|x\|_{1}\right\} \\
\geq 1-\frac{1}{n^{k}} .
\end{gathered}
$$

Proof. Since

$$
\begin{gathered}
\left\{x \in[0, \infty)^{n}:(1-\varepsilon) e^{-\gamma}<r_{n}(x)<(1+\varepsilon) e^{-\gamma}\right\} \\
=\left\{x \in[0, \infty)^{n}:\left[1-(1+\varepsilon) e^{-\gamma}\right] \frac{1}{n}\|x\|_{1}<\frac{1}{n} \sum_{i=1}^{n} x_{i}-\prod_{i=1}^{n} x_{i}^{1 / n}<\left[1-(1-\varepsilon) e^{-\gamma}\right] \frac{1}{n}\|x\|_{1}\right\},
\end{gathered}
$$

the result follows from Corollary 2.3 together with Theorem 4.1 (expressed in terms of an exponential distribution rather than normalized surface area on $\mathbb{S}_{1}^{n-1}$ ).

A result analogous to the previous one can be stated for the GM-AM ratio, using the following bounds due to S. S. Dragomir, cf. [5, Section 4]:

$$
r_{n}(x)^{n \alpha_{\max , n}} \leq \frac{\prod_{i=1}^{n} x_{i}^{\alpha_{i, n}}}{\sum_{i=1}^{n} \alpha_{i, n} x_{i}} \leq r_{n}(x)^{n \alpha_{\min , n}}
$$

Theorem 4.4. Let $k, \lambda, \varepsilon>0$, and for $1 \leq i \leq n$, let $x_{i} \in[0, \infty)$ be chosen independentlyaccording to an exponential distribution with parameter $\lambda$. Let $P_{n}$ denote the product probability on $[0, \infty)^{n}$ with density $\lambda^{n} e^{-\lambda\|x\|_{1}}$. Then there exists an $N=N(k, \varepsilon)$ such that for every $n \geq N$,

$$
\begin{aligned}
P_{n}\left\{(1-\varepsilon) e^{-n \alpha_{\max , n} \gamma}\right. & \left.<\frac{\prod_{i=1}^{n} x_{i, n}^{\alpha_{i}}}{\sum_{i=1}^{n} \alpha_{i, n} x_{i}}<(1+\varepsilon) e^{-n \alpha_{\min , n} \gamma}\right\} \\
& \geq 1-\frac{1}{n^{k}} .
\end{aligned}
$$

Proof. This easily follows from (33) together with Theorem 4.1, expressed in terms of an exponential distribution instead of normalized surface area on $\mathbb{S}_{1}^{n-1}$.

Additional probabilistic results regarding the GM-AM ratio for sequences of unequal weights can be found in [3]. The main difference between these results and Theorems 4.3-4.4, is that in [3] the probability distributions are chosen depending on the sequences of weights, while above, the same density $\lambda^{n} e^{-\lambda\|x\|_{1}}$ is used for all sequences of $n$ weights $\alpha_{i, n}$. 


\section{References}

[1] J. M. Aldaz, Self-improvement of the inequality between arithmetic and geometric means, Journal of Mathematical Inequalities, 3(2009), 213-216. arXiv:0807.1788.

[2] J. M. Aldaz, A refinement of the inequality between arithmetic and geometric means, Journal of Mathematical Inequalities, 2(2008), 473-477. arXiv:0811.3145.

[3] J. M. Aldaz, Concentration of the ratio between the geometric and arithmetic means, Journal of Theoretical Probability, 23, 498-508, DOI: 10.1007/s10959-009-0215-9. arXiv:0807.4832.

[4] J. M. Aldaz, A stability version of Hölder's inequality, Journal of Mathematical Analysis and Applications, 343(2008), 842-852. doi:10.1016/j.jmaa.2008.01.104. arXiv:0710.2307.

[5] Sever S. Dragomir, Bounds for the normalised Jensen functional, Bull. Austral. Math. Soc., 74 (2006), 471-478.

[6] Lawrence C. Evans and Ronald F. Gariepy, Measure theory and fine properties of functions, Studies in Advanced Mathematics. CRC Press, Boca Raton, FL, 1992.

[7] Herbert Federer, Geometric measure theory. Die Grundlehren der mathematischen Wissenschaften, Band 153 Springer-Verlag New York Inc., New York 1969.

[8] Shigeru Furuichi, A refinement of the arithmetic-geometric mean inequality, arXiv:0912.5227.

[9] Ronald E. Glaser, The ratio of the geometric mean to the arithmetic mean for a random sample from a gamma distribution, J. Amer. Statist. Assoc., 71(1976), 480-487.

[10] Flavia Corina Mitroi, About the precision in Jensen-Steffensen inequality, An. Univ. Craiova Ser. Mat. Inform., 37 (2010), 73-84.

[11] J. Michael Steele, The Cauchy-Schwarz master class. An introduction to the art of mathematical inequalities, MAA Problem Books Series. Mathematical Association of America, Washington, DC; Cambridge University Press, Cambridge, 2004.

Departamento de Matemáticas, Universidad Autónoma de Madrid, Cantoblanco 28049, Madrid, Spain.

E-mail: jesus.munarriz@uam.es 